\title{
New exact solutions to a KdV equation with drifting using multiple $G^{\prime} / G$-expansion schemes
}

\author{
Yinghui He, Yao Long* \\ Department of Mathematics, Honghe University, Mengzi, Yunnan, 661100, China \\ *Corresponding author, e-mail: yaolong04@163.com
}

Received 14 May 2014

Accepted 16 Jun 2016

\begin{abstract}
A KdV equation with drifting describes solitary waves propagating on an interface (liquid-air) with wave motion induced by a harmonic forcing. In this work, exact travelling wave solutions of this equation are studied using the $G^{\prime} / G$-expansion scheme and its variants. Many new exact travelling wave solutions can easily be derived from the general results under certain conditions.
\end{abstract}

KEYWORDS: nonlinear evolution equations, travelling wave solutions, solitons

\section{INTRODUCTION}

Nonlinear evolution equations (NLEEs) have been applied in fields such as optical fibres, quantum mechanics, quantum field theory, high-energy physics, fluids dynamics, biophysics, and plasma physics. A lot of physical models have supported a wide variety of solitary wave solutions. Thus the investigation of the solitary wave solutions to NLEEs plays an important role in mathematical physics. In particular, there has been considerable interest in investigating exact travelling wave solutions of NLEEs. In the recent years, many significant direct methods have been established to find exact solutions of NLEEs such as the Painlevé expansion method $^{1}$, Bäcklund and Darboux transform ${ }^{2}$, solitary wave ansatz method ${ }^{3}$, homogeneous balance method $^{4}$, Jacobi elliptic function method $^{5}$, and the $F$-expansion method ${ }^{6}$.

The $G^{\prime} / G$-expansion method proposed by Wang $\mathrm{Ml}^{7}$, is one of the most effective direct methods to obtain travelling wave solutions of NLEEs ${ }^{8-10}$. This method can also be applied to fractional ODEs ${ }^{11}$. To extend the range of its applicability, the generalized $G^{\prime} / G$-expansion method ${ }^{12}$, the extended $G^{\prime} / G$-expansion method ${ }^{13}$ and the improved $G^{\prime} / G$ expansion method ${ }^{14}$ have been developed.

The nonlinear shallow water surface waves satisfy the Korteweg-de Vries (KdV) equation

$$
w_{t}+c_{0} w_{x}+a_{1} w w_{x}+a_{2} w_{x x x}=0
$$

This equation is only valid for long waves. Solitary waves in film flows were studied by $\mathrm{Liu}^{15}$ and Heining ${ }^{16}$. These flows also show a transition to turbulence. This process is better understood if the dynamics of nonlinear waves are traceable. Rees ${ }^{17}$ derived a new NLEE for waves propagating on a liquid-air interface driven by a horizontal harmonic forcing:

$$
w_{x x x}+k^{2} w_{x}+\epsilon c \lambda_{3}\left(w_{x}^{2}-w w_{x x}\right)-\frac{2 k^{2}}{c} w_{t}=0 .
$$

This equation is more nonlinear than (1). The nonlinear term $w w_{x}$ in (1) does not appear in (2), and the reconstruction of this term from $\left(w_{x}^{2}-w w_{x x}\right)$ is not possible by order approximation equivalences. The nonlinear term in (2) is also steeper. Exact travelling wave solutions of (2) have not been reported. The aim of this paper is to derive travelling wave solutions to (2) using the $G^{\prime} / G$-expansion scheme and its variants.

The organization of the paper is as follows. Firstly, a brief account of the $G^{\prime} / G$-expansion method and its variants, i.e., the generalized, improved and extended versions, for finding the travelling wave solutions of NLEEs are given. We then study (2) using these schemes.

\section{DESCRIPTION OF METHODS}

The $G^{\prime} / G$-expansion method:

Step 1: Consider a general nonlinear PDE in the form

$$
\left(u, u_{x}, u_{t}, u_{x x}, u_{x t}, \ldots\right)=0 .
$$

Using $u(x, t)=U(\xi), \xi=\alpha x+\beta t$, we can rewrite (3) as the nonlinear ODE

$$
\left(U, U^{\prime}, U^{\prime \prime}, \ldots\right)=0
$$


where the prime denotes differentiation with respect to $\xi$.

Step 2: Suppose that the solution of ODE (4) can be written as

$$
U(\xi)=\sum_{i=0}^{n} a_{i}\left(\frac{G^{\prime}}{G}\right)^{i}+\sum_{j=1}^{n} b_{j}\left(\frac{G^{\prime}}{G}\right)^{-j},
$$

or

$U(\xi)=\sum_{i=0}^{n} a_{i}\left(\frac{G^{\prime}}{G+\sigma G^{\prime}}\right)^{i}+\sum_{j=1}^{n} b_{j}\left(\frac{G^{\prime}}{G+\sigma G^{\prime}}\right)^{-j}$,

where $\sigma, a_{i}, b_{j}$ are constants to be determined later, $n$ is a positive integer, and $G=G(\xi)$ satisfies

$$
G^{\prime \prime}+\lambda G^{\prime}+\mu G=0,
$$

where $\lambda, \mu$ are real constants. The general solutions of (7) can be listed as follows. When $\Delta=\lambda^{2}-4 \mu>0$, we obtain the hyperbolic function solution of (7)

$G(\xi)=\mathrm{e}^{-(\lambda / 2) \xi}\left(A_{1} \cosh \frac{\sqrt{\Delta}}{2} \xi+A_{2} \sinh \frac{\sqrt{\Delta}}{2} \xi\right)$.

When $\Delta=\lambda^{2}-4 \mu<0$, we obtain the trigonometric function solution of (7)

$G(\xi)=\mathrm{e}^{-(\lambda / 2) \xi}\left(A_{1} \cos \frac{\sqrt{-\Delta}}{2} \xi+A_{2} \sin \frac{\sqrt{-\Delta}}{2} \xi\right)$.

When $\Delta=\lambda^{2}-4 \mu=0$, we obtain the solution of (7)

$$
G(\xi)=\mathrm{e}^{-(\lambda / 2) \xi}\left(A_{1}+A_{2} \xi\right),
$$

where $A_{1}$ and $A_{2}$ are arbitrary constants.

Step 3: Determine the positive integer $n$ by balancing the highest order derivatives and nonlinear terms in (4).

Step 4: Substituting (5) or (6) along with (7) into (4) and then setting all the coefficients of $\left(G^{\prime} / G\right)^{k}$ (for $k=1,2, \ldots$ ) of the resulting system's numerator to zero yields a set of overdetermined nonlinear algebraic equations for $c$ and $a_{i}, b_{j}$.

Step 5: Assuming that the constants $c$ and $a_{i}, b_{i}$ can be obtained by solving the algebraic equations in Step 4, substituting these constants and the known general solutions of (7) into (5) or (6), we can obtain the explicit solutions of (3) immediately.

\section{The generalized $G^{\prime} / G$-expansion method:}

In the generalized version ${ }^{12}$, one makes the ansatz for the solution $U(\xi)$ as

$$
U(\xi)=\sum_{i=0}^{n} a_{i}\left(\frac{G^{\prime}}{G}\right)^{i}+\sum_{j=1}^{n} b_{j}\left(\frac{G^{\prime}}{G}\right)^{-j},
$$

where $G=G(\xi)$ satisfies

$$
\left(G^{\prime}\right)^{2}=h_{0}+h_{1} G+h_{2} G^{2}+h_{3} G^{3}+h_{4} G^{4},
$$

where $h_{0}, h_{1}, h_{2}, h_{3}$ and $h_{4}$ are arbitrary constants to be determined later and $a_{n} b_{n} \neq 0$. Substituting (11) into (4) and using (12), we obtain a polynomial in $G^{i}, G^{\prime} G^{i}(i=1,2 \ldots)$. Equating each coefficient of the resulting polynomial to zero yields a set of algebraic equations for $a_{i}, b_{j}$ and $h_{i}$. Substituting $a_{i}, b_{i}$ and the general solutions of (12) into (11), we obtain travelling wave solutions of the nonlinear (3).

\section{The extended $G^{\prime} / G$-expansion method}

In the extended form of this method ${ }^{13}$, the solution $U(\xi)$ of (4) can be expressed as

$$
U(\xi)=a_{0}+\sum_{i=1}^{n}\left\{a_{i} g^{i}+b_{i} g^{i-1} \sqrt{\sigma\left(1+\frac{1}{\mu} g^{2}\right)}\right\},
$$

where $g \equiv G^{\prime} / G$ and $a_{0}, a_{i}, b_{i}(i=1,2, \ldots, n)$ are constants to be determined later, $\sigma= \pm 1, n$ is a positive integer, and $G=G(\xi)$ satisfies

$$
G^{\prime \prime}+\mu G=0,
$$

where $\mu$ is a constant. Substituting (13) into (4) and using (14) and collecting all terms with the same order of $\left(G^{\prime} / G\right)^{k}$ and $\left(G^{\prime} / G\right)^{k} \sqrt{\sigma\left(1+(1 / \mu)\left(G^{\prime} / G\right)^{2}\right)}$ together, and then equating each coefficient of the resulting polynomial to zero yields a set of algebraic equations for $\mu, a_{0}, a_{i}, b_{i}(i=1, \ldots, n)$. On solving these algebraic equations, we obtain the values of the constants $\mu, a_{0}, a_{i}, b_{i}(i=1, \ldots, n)$ and then substituting these constants and the known general solutions of (14), which can be obtained by setting $\lambda=0$ in (8)-(10), into (13), we obtain the explicit solutions of nonlinear differential (3).

\section{NEW EXACT SOLUTIONS TO KdV EQUATION WITH DRIFTING}

Making the transformation $w(x, t)=\phi(\xi)$ with $\xi=$ $\alpha x+\beta t$, (2) reduces to

$\alpha^{3} \phi^{\prime \prime \prime}+k^{2} \alpha \phi^{\prime}+\epsilon c \lambda_{3}\left(\alpha^{2} \phi^{\prime 2}-\alpha^{2} \phi \phi^{\prime \prime}\right)-\frac{2 k^{2} \beta}{c} \phi^{\prime}=0$ 
where $\alpha, \beta, k, c, \epsilon$, and $\lambda_{3}$ are non-zero constants. Balancing $\phi^{\prime \prime \prime}$ and $\phi \phi^{\prime \prime}$ in (15), we obtain $n+3=$ $n+n+2$ which gives $n=1$.

\section{Using the $G^{\prime} / G$-expansion method}

Suppose that (15) have solutions in the form

$$
\phi(\xi)=a_{0}+a_{1} \frac{G^{\prime}}{G}+b_{1}\left(\frac{G^{\prime}}{G}\right)^{-1} .
$$

Substituting (16) along with (7) into (15) and then setting all the coefficients of $\left(G^{\prime} / G\right)^{k}$ for $k=$ $0,1, \ldots$ of the resulting system's numerator to zero yields a set of over-determined nonlinear algebraic equations about $a_{0}, a_{1}, b_{1}, \alpha, \beta$. Solving the overdetermined algebraic equations we obtain

$$
\begin{aligned}
& a_{0}=\frac{\alpha^{2} c^{2} \epsilon b_{1} \lambda_{3}+\alpha^{3} c \lambda^{2}+\alpha c k^{2}-2 k^{2} \beta}{\alpha^{2} c^{2} \lambda \epsilon \lambda_{3}}, \\
& a_{1}=0, b_{1}=b_{1}, \alpha=\alpha, \beta=\beta, \lambda=\lambda, \mu=0,
\end{aligned}
$$

where $\lambda \neq 0$. Otherwise

$$
\begin{array}{r}
a_{0}=\frac{\lambda a_{1}}{2}, b_{1}=0, \alpha=-\frac{c \epsilon \lambda_{3} a_{1}}{6}, \mu=0, \\
\beta=\frac{c^{2} \epsilon a_{1} \lambda_{3}\left(c^{2} \lambda^{2} \epsilon^{2} a_{1}{ }^{2} \lambda_{3}{ }^{2}-18 k^{2}\right)}{216 k^{2}} . \\
a_{1}=0, b_{1}=\frac{k^{2}(2 \beta-\alpha c)}{\alpha^{2} c^{2} \epsilon \lambda_{3}}, \lambda=0, \mu=0 .
\end{array}
$$

When $\mu=0, \lambda \neq 0$, (7) has the solution

$$
G(\xi)=A_{1}+A_{2} \mathrm{e}^{-\lambda \xi},
$$

where $A_{1}$ and $A_{2}$ are arbitrary constants. Substituting (17) and (18) into (16) and using (20), we can obtain exponential function solutions of (2) as follows:

$$
w(x, t)=\frac{\alpha^{3} c \lambda^{2}+\alpha c k^{2}-2 k^{2} \beta}{\alpha^{2} c^{2} \lambda \epsilon \lambda_{3}}-\frac{b_{1} A_{1}}{\lambda A_{2}} \mathrm{e}^{\lambda \xi},
$$

where $\xi=\alpha x+\beta t$ and

$$
w(x, t)=a_{0}+a_{1} \frac{G^{\prime}}{G}=\frac{\lambda a_{1}}{2}-\frac{a_{1} A_{2} \lambda \mathrm{e}^{-\lambda \xi}}{A_{1}+A_{2} \mathrm{e}^{-\lambda \xi}},
$$

where $\xi=\alpha x+\beta t, \alpha$ and $\beta$ are determined in (18). When $\lambda=0, \mu=0$, (7) has the solution

$$
G(\xi)=A_{1} \xi+A_{2},
$$

where $A_{1}$ and $A_{2}$ are arbitrary constants.
Substituting (18) and (19) into (16) and using (21), we obtain rational function solutions

$$
w(x, t)=a_{1} \frac{G^{\prime}}{G}=\frac{a_{1} A_{1}}{A_{1} \xi+A_{2}},
$$

where $\xi=-\frac{1}{6} c \epsilon \lambda_{3} a_{1} x-\frac{1}{12} c^{2} \epsilon \lambda_{3} a_{1} t$ and

$$
w(x, t)=a_{0}+\frac{k^{2}(2 \beta-\alpha c)}{\alpha^{2} c^{2} \epsilon \lambda_{3}}\left(\xi+\frac{A_{2}}{A_{1}}\right),
$$

where $\xi=\alpha x+\beta t$.

Suppose that (2) has the solutions in the form

$$
\phi(\xi)=a_{0}+a_{1} \frac{G^{\prime}}{G+\sigma G^{\prime}}+b_{1}\left(\frac{G^{\prime}}{G+\sigma G^{\prime}}\right)^{-1},
$$

where $G=G(\xi)$ satisfies (14). Substituting (22) along with (14) into (15) and then setting all the coefficients of $\left(G^{\prime} / G\right)^{k}$ for $k=0,1, \ldots$ of the resulting system's numerator to zero yields a set of over-determined nonlinear algebraic equations about $a_{0}, a_{1}, b_{1}, \alpha, \beta$. Solving the over-determined algebraic equations we obtain

$$
\begin{gathered}
a_{1}=-\frac{a_{0}\left(\mu \sigma^{2}+1\right)}{\mu \sigma}, b_{1}=0, \alpha=\frac{a_{0} c \epsilon \lambda_{3}}{6 \mu \sigma}, \\
\beta=\frac{a_{0} c^{2} \epsilon \lambda_{3}\left(2 a_{0}^{2} c^{2} \epsilon^{2} \lambda_{3}^{2}+9 k^{2} \mu \sigma^{2}\right)}{108 \mu^{2} \sigma^{3} k^{2}},
\end{gathered}
$$

where $\sigma \neq 0$. Otherwise

$$
\begin{gathered}
a_{0}=-\sigma b_{1}, a_{1}=0, \alpha=\frac{b_{1} c \epsilon \lambda_{3}}{6 \mu}, \\
\beta=\frac{b_{1} c^{2} \epsilon \lambda_{3}\left(2 b_{1}^{2} c^{2} \epsilon^{2} \lambda_{3}{ }^{2}+9 k^{2} \mu\right)}{108 \mu^{2} k^{2}} .
\end{gathered}
$$

Substituting (23) and (24) into (22) and using solutions of (14) which can be obtained by setting $\lambda=0$ in (8)-(10), we can obtain exact solutions of (2) as follows. When $\mu>0$, we obtain the trigonometric function solutions

$$
w(x, t)=a_{0}-\frac{a_{0}\left(\mu \sigma^{2}+1\right)}{\mu \sigma} \frac{G^{\prime}}{G+\sigma G^{\prime}}
$$

where $G=A_{1} \cos (\sqrt{\mu} \xi)+A_{2} \sin (\sqrt{\mu} \xi), \xi=\alpha x+\beta t$, $\alpha$ and $\beta$ are determined in (23) and

$$
\begin{aligned}
& w(x, t)=-\sigma b_{1}+b_{1} \frac{G+\sigma G^{\prime}}{G^{\prime}}=b_{1} \frac{G}{G^{\prime}} \\
& =b_{1} \frac{A_{1} \cos (\sqrt{\mu} \xi)+A_{2} \sin (\sqrt{\mu} \xi)}{\sqrt{\mu}\left(-A_{1} \sin (\sqrt{\mu} \xi)+A_{2} \cos (\sqrt{\mu} \xi)\right)},
\end{aligned}
$$

where $\xi=\alpha x+\beta t, \alpha$ and $\beta$ are determined in (24). 
It is easy to see that the trigonometric function solution (25) can be rewritten when $A_{1}^{2}+A_{2}^{2} \neq 0$ as

$$
w(x, t)=\frac{b_{1}}{\sqrt{\mu}} \tan \left(\sqrt{\mu} \xi+\xi_{0}\right)
$$

where $\xi_{0}=\tan ^{-1}\left(A_{1} / A_{2}\right)$. When $\mu<0$, we obtain the hyperbolic function solutions

$$
w(x, t)=a_{0}+\frac{a_{0}\left(\mu \sigma^{2}+1\right)}{\mu \sigma} \frac{G^{\prime}}{G+\sigma G^{\prime}}
$$

where $G=A_{1} \cosh (\sqrt{-\mu} \xi)+A_{2} \sinh (\sqrt{-\mu} \xi), \quad \xi=$ $\alpha x+\beta t, \alpha$ and $\beta$ are determined in (23) and

$$
\begin{aligned}
& w(x, t)=-\sigma b_{1}+b_{1} \frac{G+\sigma G^{\prime}}{G^{\prime}}=b_{1} \frac{G}{G^{\prime}} \\
& =\frac{b_{1}\left(A_{1} \cosh (\sqrt{-\mu} \xi)+A_{2} \sinh (\sqrt{-\mu} \xi)\right)}{\sqrt{-\mu}\left(A_{1} \sinh (\sqrt{-\mu} \xi)+A_{2} \cosh (\sqrt{-\mu} \xi)\right)},
\end{aligned}
$$

where $\xi=\alpha x+\beta t, \alpha$ and $\beta$ are determined in (24). It is easy to see that the hyperbolic function solution (26) can be rewritten as

$$
w(x, t)=\frac{b_{1}}{\sqrt{-\mu}} \tanh \left(\sqrt{-\mu} \xi+\xi_{0}\right),
$$

where $\xi_{0}=\tanh ^{-1}\left(A_{1} / A_{2}\right)$ when $A_{1}{ }^{2}<A_{2}^{2}$ and as

$$
w(x, t)=\frac{b_{1}}{\sqrt{-\mu}} \operatorname{coth}\left(\sqrt{-\mu} \xi+\xi_{0}\right),
$$

where $\xi_{0}=\operatorname{coth}^{-1}\left(A_{1} / A_{2}\right)$ when ${A_{1}}^{2}>A_{2}^{2}$.

\section{Using the generalized $G^{\prime} / G$-expansion method}

Suppose that (15) has solutions in the form

$$
\phi(\xi)=a_{0}+a_{1} \frac{G^{\prime}}{G}+b_{1}\left(\frac{G^{\prime}}{G}\right)^{-1} .
$$

In this case, $G=G(\xi)$ satisfies the Jacobi elliptic (12). Substituting (27) along with (12) into (15) and then setting all the coefficients of $G^{i}, G^{\prime} G^{i}(i=$ $1,2 \ldots)$ of the resulting system's numerator to zero, yields a set of over-determined nonlinear algebraic equations about $a_{0}, a_{1}, b_{1}, \alpha, \beta$. Solving the overdetermined algebraic equations, we can obtain the following results:

$$
\begin{aligned}
& a_{0}=0, a_{1}=0, h_{0}=0, h_{1}=0, h_{4}=\frac{h_{3}^{2}}{4 h_{2}}, \\
& \beta=\frac{\alpha c\left(-\alpha b_{1} c \epsilon \lambda_{3}+\alpha^{2} h_{2}+k^{2}\right)}{2 k^{2}} . \\
& a_{0}=0, a_{1}=0, h_{2}=\frac{h_{1}^{2}}{4 h_{0}}, h_{3}=0, h_{4}=0, \\
& \beta=\frac{\alpha c\left(4 \alpha b_{1} c \epsilon h_{0} \lambda_{3}+\alpha^{2} h_{1}{ }^{2}+4 k^{2} h_{0}\right)}{8 k^{2} h_{0}} .
\end{aligned}
$$

Considering (28), (12) becomes

$$
\left(G^{\prime}\right)^{2}=h_{2} G^{2}+h_{3} G^{4}+\frac{h_{3}^{2}}{4 h_{2}} G^{4} .
$$

The general solutions of (30) are

$$
\begin{aligned}
& G_{1}(\xi)=\frac{-h_{2}}{h_{3}}\left(1 \pm \operatorname{coth}\left(\frac{\sqrt{h_{2}}}{2} \xi+\xi_{0}\right)\right), \\
& G_{2}(\xi)=\frac{-h_{2}}{h_{3}}\left(1 \pm \tanh \left(\frac{\sqrt{h_{2}}}{2} \xi+\xi_{0}\right)\right),
\end{aligned}
$$

where $h_{2}>0, \xi_{0}$ are arbitrary constants.

Substituting (28) into (27) and making use of (31) and (32), we can obtain hyperbolic function solutions of (2).

$$
\begin{aligned}
& w(x, t)=\frac{-2 b_{1}}{\sqrt{h_{2}}\left(\operatorname{coth}\left(\frac{\sqrt{h_{2}}}{2} \xi+\xi_{0}\right) \pm 1\right)}, \\
& w(x, t)=\frac{-2 b_{1}}{\sqrt{h_{2}}\left(\tanh \left(\frac{\sqrt{h_{2}}}{2} \xi+\xi_{0}\right) \pm 1\right)},
\end{aligned}
$$

where $\xi=\alpha x+\left(\alpha c\left(-\alpha b_{1} c \epsilon \lambda_{3}+\alpha^{2} h_{2}+k^{2}\right) / 2 k^{2}\right) t$.

Considering (29), (12) becomes

$$
\left(G^{\prime}\right)^{2}=h_{0}+h_{1} G+\frac{h_{1}^{2}}{4 h_{0}} G^{2} .
$$

The general solutions of (33) are

$$
G(\xi)=\frac{C \mathrm{e}^{ \pm h_{1} / 2 \sqrt{h_{0} \xi}}-2 h_{0}}{h_{1}}
$$

where $h_{0}>0, C$ are arbitrary constants.

Substituting (29) into (27) and making use of (34), we can obtain exponential function solutions of (2):

$$
w(x, t)= \pm \frac{2 b_{1} \sqrt{h_{0}}}{h_{1}} \mp \frac{4 b_{1} \sqrt{h_{0}^{3}}}{h_{1} C} \mathrm{e}^{\mp\left(h_{1} / 2 \sqrt{h_{0}}\right) \xi},
$$

where $\xi=\alpha x+\left(\alpha c\left(4 \alpha b_{1} c \epsilon h_{0} \lambda_{3}+\alpha^{2} h_{1}{ }^{2}+\right.\right.$ $\left.\left.4 k^{2} h_{0}\right) / 8 k^{2} h_{0}\right) t$.

Remark 1 Besides (28) and (29), we can also obtain many other cases. Because of the similarities with the results discussed in the previous section, we do not list them in detail. 


\section{Using the extended $G^{\prime} / G$-expansion method}

Suppose that (15) has solutions in the form

$$
\phi(\xi)=a_{0}+a_{1} \frac{G^{\prime}}{G}+b_{1} \sqrt{\sigma\left(1+\frac{1}{\mu}\left(\frac{G^{\prime}}{G}\right)^{2}\right)},
$$

where $a_{0}, a_{1}, b_{1}$ are constants to be determined later, $\sigma= \pm 1, n$ is a positive integer, and $G=G(\xi)$ satisfies the second order linear ODE (14).

Substituting (35) along with (14) into (15) and then setting all the coefficients of $\left(G^{\prime} / G\right)^{k}$ and $\left(G^{\prime} / G\right)^{k} \sqrt{\sigma\left(1+(1 / \mu)\left(G^{\prime} / G\right)^{2}\right)}(k=0,1, \ldots)$ of the resulting system to zero yields a set of overdetermined nonlinear algebraic equations about $a_{0}$, $a_{1}, b_{1}, \alpha, \beta$. Solving the over-determined algebraic equations, we can obtain the following results:

$$
\begin{aligned}
& a_{0}=0, a_{1}=\frac{-3 \alpha}{c \epsilon \lambda_{3}}, b_{1}= \pm \frac{3 \alpha}{c \epsilon \lambda_{3}} \sqrt{\frac{\mu}{\sigma}}, \\
& \alpha=\alpha, \beta=\frac{c \alpha\left(2 \alpha^{2} \mu+k^{2}\right)}{2 k^{2}},
\end{aligned}
$$

where $\sigma \mu>0$. Otherwise

$$
a_{0}=0, a_{1}=\frac{-6 \alpha}{c \in \lambda_{3}}, b_{1}=0, \beta=\frac{8 \alpha^{3} c \mu+\alpha c k^{2}}{2 k^{2}} .
$$

Using (36) and the general solutions of (14), we can find the following travelling wave solutions of (2). When $\mu<0, \sigma=-1$, we have the hyperbolic function solution

$$
\begin{aligned}
& w(x, t)=a_{1} \frac{G^{\prime}}{G}+b_{1} \sqrt{\sigma\left(1+\frac{1}{\mu}\left(\frac{G^{\prime}}{G}\right)^{2}\right)} \\
& =\frac{-3 \alpha \sqrt{-\mu}}{c \epsilon \lambda_{3}}\left\{\frac{A_{1} \sinh (\sqrt{-\mu} \xi)+A_{2} \cosh (\sqrt{-\mu} \xi)}{A_{1} \cosh (\sqrt{-\mu} \xi)+A_{2} \sinh (\sqrt{-\mu} \xi)}\right. \\
& \pm \sqrt{-1+\left(\frac{A_{1} \sinh (\sqrt{-\mu} \xi)+A_{2} \cosh (\sqrt{-\mu} \xi)}{A_{1} \cosh (\sqrt{-\mu} \xi)+A_{2} \sinh (\sqrt{-\mu} \xi)}\right)^{2}},
\end{aligned}
$$

where $\xi=\alpha x+\left(c \alpha\left(2 \alpha^{2} \mu+k^{2}\right) / 2 k^{2}\right) t$. In particular, setting $A_{1} \neq 0, A_{2}=0$, then (38) can be written as

$w(x, t)=\frac{-3 \alpha \sqrt{-\mu}}{c \epsilon \lambda_{3}}(\tanh (\sqrt{-\mu} \xi) \pm \operatorname{isech}(\sqrt{-\mu} \xi))$.

Setting $A_{1}=0, A_{2} \neq 0$, then (38) can be written as

$w(x, t)=\frac{-3 \alpha \sqrt{-\mu}}{c \in \lambda_{3}}(\operatorname{coth}(\sqrt{-\mu} \xi) \pm \operatorname{csch}(\sqrt{-\mu} \xi))$.

When $\mu>0, \sigma=1$, we have the trigonometric function solution

$$
\begin{aligned}
& w(x, t)=a_{1} \frac{G^{\prime}}{G}+b_{1} \sqrt{\sigma\left(1+\frac{1}{\mu}\left(\frac{G^{\prime}}{G}\right)^{2}\right)} \\
& =\frac{-3 \alpha \sqrt{\mu}}{c \epsilon \lambda_{3}}\left\{\frac{-A_{1} \sin (\sqrt{\mu} \xi)+A_{2} \cos (\sqrt{\mu} \xi)}{A_{1} \cos (\sqrt{\mu} \xi)+A_{2} \sin (\sqrt{\mu} \xi)}\right. \\
& \left. \pm \sqrt{1+\left(\frac{-A_{1} \sin (\sqrt{\mu} \xi)+A_{2} \cos (\sqrt{\mu} \xi)}{A_{1} \cos (\sqrt{\mu} \xi)+A_{2} \sin (\sqrt{\mu} \xi)}\right)^{2}}\right\}
\end{aligned}
$$

where $\xi=\alpha x+\left(c \alpha\left(2 \alpha^{2} \mu+k^{2}\right) / 2 k^{2}\right) t$. In particular, setting $A_{1} \neq 0, A_{2}=0$, then (39) can be written as

$$
w(x, t)=\frac{3 \alpha \sqrt{\mu}}{c \in \lambda_{3}}(-\tan (\sqrt{\mu} \xi) \pm \sec (\sqrt{\mu} \xi)),
$$

setting $A_{1}=0, A_{2} \neq 0$, then (39) can be written as

$$
w(x, t)=\frac{3 \alpha \sqrt{\mu}}{c \epsilon \lambda_{3}}(\cot (\sqrt{\mu} \xi) \pm \csc (\sqrt{\mu} \xi)) .
$$

Using (37) and the general solutions of (14), we can find the exact travelling wave solutions of (2). The process is similar to (36). Hence we omit it for convenience. Note that $\sigma$ can be a non-zero constant.

The correctness of all the solutions is verified by substituting them into (2).

Acknowledgements: This study is supported by the Natural Science Foundation of China (Nos. 11461022, 11161020 and 11361023), Science Foundation of Yunnan province (2014FA037) and Middle-Aged Academic Backbone of Honghe University (No. 2014GG0105).

\section{REFERENCES}

1. Weiss J, Tabor M, Carnevale G (1983) The Painlevé property for partial differential equations. $J$ Math Phys 24, 522-6.

2. Matveev VB, Salle MA (1991) Darboux Transformations and Solitons, Springer, Berlin.

3. Triki H, Crutcher S, Yildirim A, Hayat T, Aldossary OM, Biswas A (2012) Bright and dark solitons of the modified complex Ginzburg Landau equation with parabolic and dual-power law nonlinearity. Rom Rep Phys 64, 367-80.

4. Wang M, Zhou Y, Li Z (1996) Application of a homogeneous balance method to exact solutions of nonlinear equations in mathematical physics. Phys Lett A 216, 67-75.

5. Bhrawy AH, Abdelkawy MA, Biswas A (2013) Cnoidal and snoidal wave solutions to coupled nonlinear wave equations by the extended Jacobi's elliptic function method. Comm Nonlin Sci Numer Simulat 18, 915-25. 
6. Ebadi G, Fard NY, Bhrawy AH, Kumar S, Triki H, Yildirim A, Biswas A (2013) Solitons and other solutions to the $(3+1)$-dimensional extended KadomtsevPetviashvili equation with power law nonlinearity. Rom Rep Phys 65, 27-62.

7. Wang MX, Li XZ, Zhang JL (2008) The $G^{\prime} / G$ expansion method and traveling wave solutions of nonlinear evolution equations in mathematical physics. Phys Lett A 372, 417-23.

8. Sardar A, Husnine SM, Rizvi STR, Younis M, Ali K (2015) Multiple traveling wave solutions for electrical transmission line model. Nonlinear Dynam 82, 1317-24.

9. Younis M, Rizvi STR, Zhou Q, Biswas A, Belic M (2015) Optical solitons in dual-core fibers with G'/Gexpansion scheme. J Optoelectronics Adv Mater 17, 505-10.

10. Younis M, ur Rehman H, Iftikhar M (2014) Traveling wave solutions to some time-space nonlinear evolution equations. Appl Math Comput 249, 81-8.

11. Bekir A, Güner Ö (2013) Exact solutions of nonlinear fractional differential equations by $G^{\prime} / G$-expansion method. Chin Phys B 22, 110202.

12. Jabbari A, Heris JM, Kheiri H, Bekir A (2014) A generalization of $G^{\prime} / G$-expansion method and its application to nonlinear reaction-diffusion equations arising in mathematical biology. Int J Biomathematics 7, 1450025.

13. Guo SM, Zhou YB (2010) The extended $G^{\prime} / G$ expansion method and its applications to the Whitham-Broer-Kaup-Like equations and coupled Hirota-Satsuma KdV equations. Appl Math Comput 215, 3214-21.

14. Naher H, Abdullah FA, Bekir A (2012) Abundant traveling wave solutions of the compound KdV-Burgers equation via the improved $G^{\prime} / G$-expansion method. AIP Adv 2, 042163.

15. Liu J, Gollub JP (1994) Solitary wave dynamics of film flows. Phys Fluids 6, 1702-12.

16. Heining C, Pollak T, Aksel N (2012) Pattern formation and mixing in three-dimensional film flow. Phys Fluids 24, 042102.

17. Rees JM, Zimmerman WB (2011) An intermediate wavelength, weakly nonlinear theory for the evolution of capillary gravity waves. Wave Motion 8, 707-16. 